\title{
Generic pathways to stability in concentrated protein mixtures $\dagger$
}

\author{
Ilja K. Voets, ${ }^{* a b}$ Veronique Trappe $^{c}$ and Peter Schurtenberger ${ }^{b d}$
}

We present a series of experimental results that disclose the crucial role of ionic strength and partial volume fractions in the control of the phase behaviour of binary protein mixtures. Our findings can be understood as that the ionic strength determines the relative contribution of the entropy of the protein counter-ions to the overall thermodynamics of the system. Associative phase separation and crystallization observed at, respectively, low and high ionic strength are suppressed at intermediate salt concentrations, where the entropy gain upon releasing the counter-ions from the double layer of the proteins is negligible and the entropy loss upon confining the counter-ions within the protein crystal phase significant. Moreover, we find that the partial volume fraction of the protein prone to crystallize determines the crystallization boundary and that the presence of other proteins strongly delays crystallization, leading to temporarily stable mixtures. These findings suggest that stability in more complex protein mixtures, such as the cytosol, relates to the ionic strength and protein composition rather than to protein specific properties.

\section{Introduction}

Concentrated protein mixtures are highly susceptible to various phase transitions, such as crystallization, liquid-liquid phase separation, and associative phase separation; the latter referring to the complexation of oppositely charged proteins resulting in coexisting protein-rich and protein-poor phases. ${ }^{1-5}$ Despite this tendency to phase separate, numerous examples of stable multicomponent protein mixtures exist in nature. ${ }^{6-8}$ The intracellular fluid of living cells, also known as the cytosol, is perhaps the most prominent example. It contains $200-350 \mathrm{mg} \mathrm{ml}^{-1}$ of thousands of different proteins. ${ }^{9,10}$ The primary requirement for its functionality is stability, i.e. the proteins remain dispersed. Despite the obvious importance of this stability, there are surprisingly few studies devoted to its origin, the existing work calling upon diverse arguments. ${ }^{11-14}$ Indeed, while Doye and Poon propose that proteins specifically evolved to expose amino acids at their surface that disfavour the formation of crystal contacts, ${ }^{13,14}$ Sear et al. argue that protein interactions eventually cancel out if the number of components within the

\footnotetext{
${ }^{a}$ Institute for Complex Molecular Systems and Laboratory of Macromolecular and Organic Chemistry, Eindhoven University of Technology, P.O. Box 513, 5600 MB Eindhoven,

mixture becomes large enough. ${ }^{11,12}$ Our work exposes pathways to stability that require neither specific surface properties ${ }^{13,14}$ nor multi-type interactions, ${ }^{11,12}$ denoting instead the ionic strength and the partial volume fraction of a given protein species as the key parameters controlling the stability of mixtures.

\section{Experimental}

\section{Materials}

The hen egg-white lysozyme used in this work is purchased from Sigma (L6876, LOT 117K1547); $\alpha$-lactalbumin is a kind gift of Davisco Foods. Stock solutions of both proteins are prepared by dissolution of the protein powders into a $20 \mathrm{mM}$ HEPES buffer at $\mathrm{pH}=7.8$ and varying sodium chloride concentrations, $c_{\mathrm{NaCl}}=0,100,200,300,400,500 \mathrm{mM}$. The initial overall protein concentrations of $C_{i} \approx 100 \mathrm{~g} \mathrm{l}^{-1}$ are determined by weight and correspond to an overall protein volume fraction of $\varphi \approx 0.07$, assuming a protein density of $\rho=1.351 \mathrm{~g} \mathrm{~cm}^{-3}$. Undissolved material is removed by filtration over a $0.22 \mu \mathrm{m}$ Millex-GV filter, such that the actual initial protein concentration $c_{i^{\prime}}$ is $8-15 \%$ lower than $C_{i}$, as determined by UV-Vis spectroscopy. Mixtures are prepared by mixing the stock solutions, equilibrated for one hour and centrifuged for $1 \mathrm{~h}$ at $10000 \mathrm{rpm}$. Subsequently they are left to equilibrate at room temperature, $T \approx 22{ }^{\circ} \mathrm{C}$, and after a given equilibration time again submitted to a $1 \mathrm{~h}$ centrifugation step at $10000 \mathrm{rpm}$; the protein concentration in the supernatant is then determined by UV-Vis spectroscopy. Labelling of lysozyme with rhodamine (RBITC) and $\alpha$-lactalbumin with fluorescein (FITC) is done following the procedure of Gorbenko et al. ${ }^{15}$ 


\section{UV-Vis spectroscopy}

Protein concentrations are determined by UV-Vis experiments at $280 \mathrm{~nm}$ using mole fraction weighted extinction coefficients $\varepsilon$. We use $\varepsilon_{\text {mix }}=f_{\alpha} \varepsilon_{\alpha}+f_{\text {LZ } \varepsilon_{\text {LZ }}}$ for our $\alpha$-lactalbumin/lysozyme mixtures with, respectively, $\varepsilon_{\alpha}=28514$ and $\varepsilon_{\mathrm{LZ}}=377521 \mathrm{~mol}^{-1} \mathrm{~cm}^{-1}$ for pure $\alpha$-lactalbumin and lysozyme solutions.

\section{Optical and confocal microscopy}

Bright field images are taken with a Leica DM IRB inverted microscope. Fluorescence resolved images are taken with a Leica TCS SP5 confocal set-up consisting of an inverted DMI $6000 \mathrm{~B}$ microscope and motorized stage. Fluorescence is excited at $488 \mathrm{~nm}$ (FITC) and 543 (RBITC) and observed at 537-551 nm (FITC) and 660-700 nm (RBITC).

\section{Results and discussion}

Aiming to rigorously test the stability of concentrated protein mixtures with respect to phase separation, we chose a system that is particularly prone to exhibit associative phase separation and crystallization: $\alpha$-lactalbumin/lysozyme mixtures. These secretory proteins are similar in mass and size and at the $\mathrm{pH}$ of $7.8 \pm 0.1$ used in our experiments they carry a net charge of equal magnitude but opposite sign. ${ }^{16,17}$ These conditions facilitate associative phase separation. The means to also stringently test stability with respect to crystallization is given by the strong tendency of lysozyme to crystallize. We investigate the phase behaviour of these mixtures at varying sodium chloride concentrations, $0 \leq c_{\mathrm{NaCl}} \leq 500 \mathrm{mM}$, and varying protein compositions, while maintaining the overall protein concentration fixed at $c_{i^{\prime}}=88 \pm 4 \mathrm{~g}^{-1}$ (see the Experimental section). After a given equilibration time $t_{\mathrm{eq}}$ at a temperature of $T \approx 22{ }^{\circ} \mathrm{C}$ the mixtures are centrifuged, where we find that some mixtures phase separated in a turbid protein-rich phase that sedimented to the bottom of the container, while the protein-poor phase remained in the supernatant. We quantify the amount of protein in the supernatant $c_{\mathrm{sn}}$ using UV-Vis spectroscopy, where $c_{\mathrm{sn}}=c_{i^{\prime}}$ for systems, which did not exhibit any phase separation.

As shown for systems after $t_{\text {eq }} \sim 2$ months in Fig. 1a, the dependence of $c_{\mathrm{sn}}$ on the lysozyme mole fraction $f_{\mathrm{Lz}}$ exhibits characteristics that distinctly depend on salt concentration. For $c_{\mathrm{NaCl}}=0$ and $100 \mathrm{mM}$, we observe a fairly symmetric "dip". This feature is a typical characteristic of associative phase separation that occurs stoichiometrically in mixtures of oppositely charged polymers, colloids and proteins at low ionic strength. ${ }^{18,19}$ The magnitude of the minimum decreases as the salt concentration increases, which implies that the driving force for associative phase separation becomes less effective as the ionic strength increases. Indeed, for $c_{\mathrm{NaCl}}=$ $200 \mathrm{mM}$ we find that $c_{\mathrm{sn}}$ is essentially identical to $c_{i^{\prime}}$, which indicates that at this salt concentration phase separation is at least temporarily suppressed. Upon increasing the salt concentration even further, we find that $c_{\mathrm{sn}}$ exhibits again a dependence on the protein composition. However, in contrast to the behaviour observed at low salt concentrations, the dependence of $c_{\mathrm{sn}}$ on $f_{\mathrm{Lz}}$ exhibits here a monotonic trend; $c_{\mathrm{sn}}$ decreases with increasing $f_{\mathrm{LZ}}$ beyond some critical value of $f_{\mathrm{LZ}}$. A comparison of
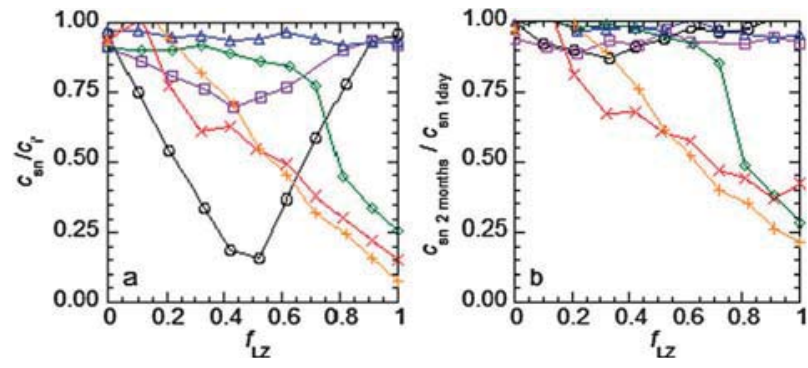

Fig. 1 (a) Protein concentration in the supernatant normalized by the protein concentration of the initial mixture, $c_{\mathrm{sn}} / c_{i^{\prime}}$, as a function of lysozyme mole fraction, $f_{\mathrm{LZ}}$. Results are obtained after an equilibration time of $\sim 2$ months. (b) Ratio of the protein concentrations in the supernatants after an equilibration time of $\sim 2$ months and 1 day. The salt concentrations are: $c_{\mathrm{NaCl}}=0 \mathrm{mM}$ (black $\bigcirc$ ), $100 \mathrm{mM}$ (purple $\square$ ), $200 \mathrm{mM}$ (blue $\triangle$ ), $300 \mathrm{mM}($ green $\diamond), 400 \mathrm{mM}($ red $\times$ ), and $500 \mathrm{mM}$ (orange + ). The lines are guides to the eye.

the behaviour for $c_{\mathrm{NaCl}}=300 \mathrm{mM}$ and $c_{\mathrm{NaCl}}=400,500 \mathrm{mM}$ reveals that this critical value shifts to lower $f_{\mathrm{Lz}}$ as the salt content is increased.

To evaluate the differences in the mechanisms leading to phase separation at, respectively, low and high ionic strength we compare the supernatant concentrations obtained after $t_{\text {eq }}$ $\sim 2$ months and $t_{\mathrm{eq}}=1$ day in Fig. 1b. This analysis reveals that while the final states are essentially reached after $t_{\mathrm{eq}}=1$ day for systems with salt concentrations of up to $200 \mathrm{mM}$, the phase separation process continuous to evolve beyond 1 day at higher salt concentrations. This strongly suggests that the phase separation process observed under these conditions is a crystallization process.

To test this we examine the optical appearance of the precipitates in bright field microscopy. As typical examples for precipitates formed at, respectively, low and high ionic strength, we show the micrographs obtained for $f_{\mathrm{LZ}}=0.7$ with $c_{\mathrm{NaCl}}=0 \mathrm{mM}$ and $c_{\mathrm{NaCl}}=500 \mathrm{mM}$ in Fig. $2 \mathrm{a}$ and $\mathrm{b}$. Clearly, while the precipitates obtained at low ionic strength appear amorphous, the precipitates obtained at high ionic strength are crystalline. To assess the composition of both we perform additional experiments using fluorescently labelled proteins, where $\alpha$-lactalbumin is labelled with fluorescein (FITC green) and lysozyme with rhodamine (RBITC red). The images obtained in confocal microscopy reveal that the amorphous phases contain both proteins; as shown for $f_{\mathrm{LZ}}=$ 0.5 and $c_{\mathrm{NaCl}}=0$ in Fig. $2 \mathrm{c}$, the colour of the precipitates is a mix of red and green in the superimposed images. By contrast, the crystalline precipitate obtained for $f_{\mathrm{Lz}}=0.5$ and $c_{\mathrm{NaCl}}=$ $500 \mathrm{mM}$ is predominantly red, which indicates that the crystals are essentially composed of pure lysozyme (Fig. 2d). These findings unambiguously demonstrate that phase separation is associative at low ionic strength and segregative at high ionic strength. The prism-like shape of the lysozyme crystals shown in Fig. 2b suggests that lysozyme crystallizes in the tetragonal form, consistent with the lysozyme crystal form found in other experiments. ${ }^{21-23}$ This would imply that the volume fraction of the condensed phase is $\varphi_{\mathrm{LZ}}=0.605$ at high ionic strength. At low ionic strength, determination of the density of the condensed phase $\varphi_{\mathrm{p}}$ is somewhat hampered due to the very small amount of the precipitate obtained. For $f_{\mathrm{LZ}}=0.5$ and $c_{\mathrm{NaCl}}=0 \mathrm{mM}$, where 

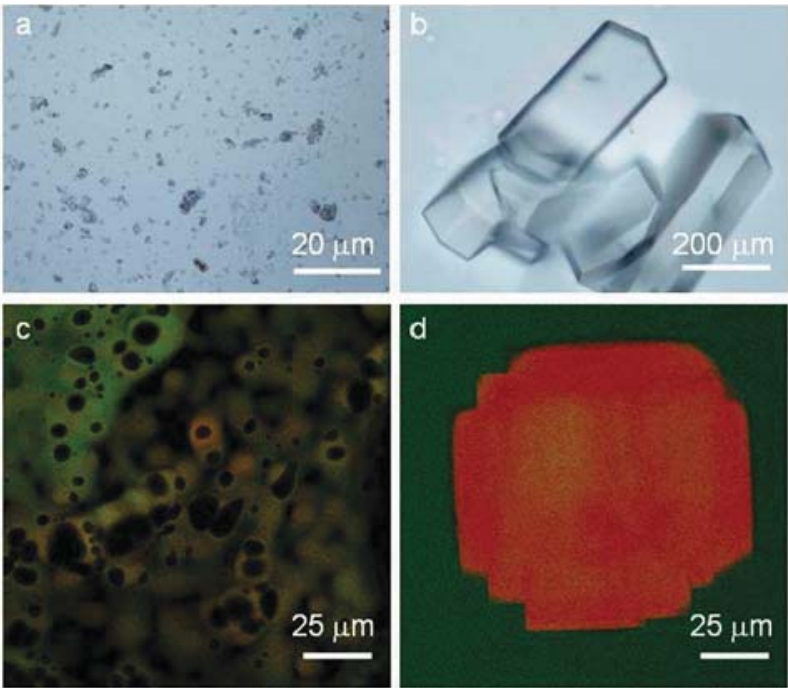

Fig. 2 (a) Optical micrographs of amorphous precipitates from $\alpha$-lactalbumin/lysozyme mixtures at $f_{\mathrm{LZ}}=0.7$ and $c_{\mathrm{NaCl}}=100 \mathrm{mM}$. (b) Optical micrographs of crystalline precipitates from mixtures at $f_{\mathrm{LZ}}=0.7$ and $c_{\mathrm{NaCl}}=500 \mathrm{mM}$. (c and d) Confocal microscopy images of (c) amorphous phases at $f_{\mathrm{LZ}}=0.5$ and $c_{\mathrm{NaCl}}=0 \mathrm{mM}$, and (d) crystalline phases at $f_{\mathrm{LZ}}=0.5$ and $c_{\mathrm{NaCl}}=500 \mathrm{mM}$ using fluorescently labelled proteins, RBITC-lysozyme (red) and FITC- $\alpha$-lactalbumin (green). The red and green images of the disordered structures observed at low ionic strength (c) nearly superimpose, indicating that the amorphous phases are composed of both, lysozyme and $\alpha$-lactalbumin. The crystalline phases obtained at high salt concentration (d) are predominantly red, indicating that the crystals essentially contain pure lysozyme.

we obtain the largest amount of precipitate, we estimate $\varphi_{\mathrm{p}}$ to be approximately 0.41 , based on the fraction of the volume occupied by the precipitate. However, it appears likely that this density will here vary depending on the conditions used, as associative phase separation leads to amorphous aggregates with internal densities that most probably will depend on the kinetics of the aggregation process.

To gain a general overview of the phase behaviour observed after $t_{\text {eq }} \sim 2$ months, we report our findings in the phase diagram shown in Fig. 3. Here we define a sample as stable (closed symbols) when $c_{\mathrm{sn}} / c_{i^{\prime}} \geq 0.9$ and as unstable (open symbols) when $c_{\mathrm{sn}} / c_{i^{\prime}}<0.9$, and we distinguish between the unstable samples where phase separation is the result of associative phase separation (open squares) and crystallization (open triangles). The phase space of instability is clearly delimited and exhibits a gap at intermediate salt concentrations. Though $\alpha$-lactalbumin and lysozyme are both secretory proteins and thus presumably only marginally model the behaviour of cytosolic proteins, it is worth noticing that the range of salt concentrations guaranteeing maximum stability is near to the physiological salt conditions in mammals. ${ }^{6}$ This suggests that the intracellular salt concentration may be a key parameter for the stability of intracellular fluids.

We can qualitatively account for the observed behaviour considering the entropic contributions of the protein counter-ions to the overall thermodynamics of the system, as recently proposed for the modelling of the thermodynamics of protein crystallization. ${ }^{24-26}$ Upon associative phase separation the counter-ions are released from the double layer of the proteins, while they are confined

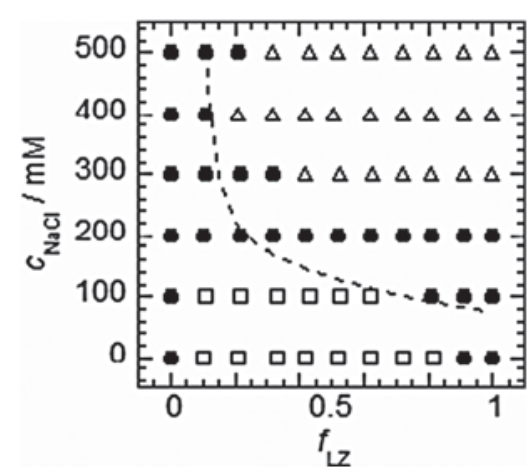

Fig. 3 Phase diagram displaying the phase behaviour of $\alpha$-lactalbumin/ lysozyme mixtures at $\mathrm{pH}=7.8$ and $T \approx 22{ }^{\circ} \mathrm{C}$ as a function of salt concentration and protein composition. The overall concentration of protein is $c_{i^{\prime}}=88 \pm 4 \mathrm{~g} \mathrm{l}^{-1}$. The data are obtained after an equilibration time of $\sim 2$ months. The stable states $(\bigcirc)$ are defined by $c_{\mathrm{sn}} / c_{i^{\prime}} \geq 0.9$. Conditions for associative phase separation are marked by $(\square)$ and the formation of lysozyme crystals by $(\triangle)$. The dashed line corresponds to the saturation limit of a pure lysozyme system (see $\mathrm{ESI} \dagger^{20}$ ).

within the crystal phase upon crystallization. ${ }^{18,19,27-29}$ The release and confinement entail, respectively, a gain and loss in the entropy of the counter-ions. However, while the gain is significant enough at low ionic strength to considerably contribute to the free energy of the system, the loss is negligible enough at high ionic strength to allow for crystallization. In between these two extremes, where neither the entropy gain upon release is significant enough nor the entropy loss upon confinement is small enough, the system is stable. Alternatively we can express this relation in terms of effective interactions that are controlled by the ionic strength. While at low ionic strength the mutual interactions are dominant, the like-like attraction becomes dominant at high ionic strength. Varying the ionic strength in a continuous manner will thus lead to a switch from mutual to like-like attraction, which implies a regime at intermediate ionic strength where neither of both is significant enough to lead to phase separation. Maintaining this intermediate ionic strength will thus guarantee stability.

To further explore the origin of stability we compare the crystallization behaviour of lysozyme in our mixtures to that of equivalent systems containing lysozyme only. For this purpose we evaluate the position of the saturation limit $S_{\mathrm{LZ}}$ of systems that contain only lysozyme under the conditions used in our experiments (see $\mathrm{ESI} \dagger^{20}$ ). This boundary shown as a dashed line in Fig. 3 closely matches the onset of crystallization in the mixtures at salt concentrations exceeding $200 \mathrm{mM}$. Indeed, assuming that the lysozyme concentration in the supernatant of the mixtures $c_{\mathrm{sn}, \mathrm{LZ}}^{\mathrm{mix}}$ at high ionic strength can be evaluated as the difference of the protein concentration in the supernatant and the overall concentration of $\alpha$-lactalbumin, $c_{\mathrm{sn}, \mathrm{LZ}}^{\mathrm{mix}}=c_{\mathrm{sn}}^{\mathrm{mix}}-c_{i^{\prime} \alpha}^{\mathrm{mix}}$, we find that $c_{\mathrm{sn}, \mathrm{L} Z}^{\mathrm{mix}}$ is in good agreement with that expected for pure lysozyme. This effectively implies that the presence of $\alpha$-lactalbumin hardly affects the crystallization of lysozyme, these systems behaving like quasi-ideal mixtures of two proteins that do not interact with one another. Note that typical protein mixtures encountered in nature contain significantly more than only two protein species, such that the likelihood of exceeding the saturation limit of any of the species is very low.

At $c_{\mathrm{NaCl}}=100$ and $200 \mathrm{mM}$, we find that none of the samples with lysozyme concentrations exceeding $S_{\mathrm{Lz}}$ crystallized during 


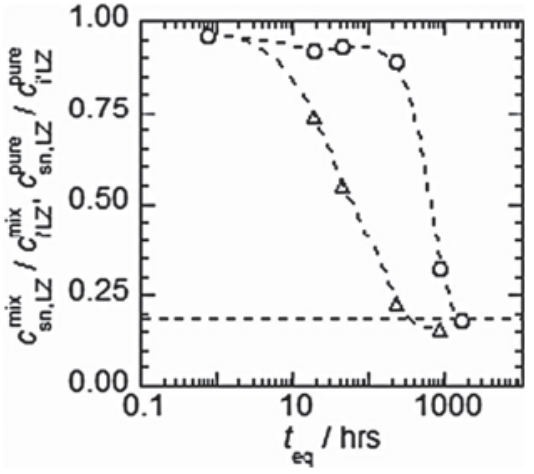

Fig. 4 Temporal evolution of lysozyme concentration in the supernatant of crystallizing samples as measured by UV-Vis spectroscopy. The triangles denote the behaviour obtained for a pure lysozyme system with an initial concentration of $c_{i^{\prime}, \mathrm{LZ}}^{\text {pure }}=55 \mathrm{~g} \mathrm{l}^{-1}$; the circles denote the behaviour obtained for a $\alpha$-lactalbumin/lysozyme-mixture with an initial lysozyme concentration of $c_{i^{\prime}, \mathrm{LZ}}^{\operatorname{mix}}=55 \mathrm{~g}^{-1}$, corresponding to $f_{\mathrm{LZ}}=0.62$ in our usual notation. In both cases the salt concentration is $c_{\mathrm{NaCl}}=400 \mathrm{mM}$. Correspondingly, the dashed line indicates the saturation limit $S_{\mathrm{LZ}}$ for pure lysozyme with $c_{\mathrm{NaCl}}=400 \mathrm{mM}$. While $c_{\mathrm{sn}, \mathrm{LZ}}^{\text {pure }}$ corresponds directly to the protein concentration in the supernatant, we evaluate $c_{\mathrm{sn}, \mathrm{LZ}}^{\mathrm{mix}}$ assuming that $\alpha$-lactalbumin remains entirely in the supernatant, such that $c_{\mathrm{sn}, \mathrm{LZ}}^{\mathrm{mix}}=c_{\mathrm{sn}}^{\mathrm{mix}}-c_{i^{\prime} \alpha}^{\operatorname{mix}}$.

the equilibration time of $\sim 2$ months; this includes the systems containing only lysozyme at $f_{\mathrm{Lz}}=1$. We can account for this considering that the nucleation rate of lysozyme decreases with decreasing salt concentration and the degree of supersaturation. ${ }^{30-35}$ Thus, even if not in equilibrium, systems with lysozyme concentrations exceeding $S_{\mathrm{Lz}}$ can be regarded as temporarily stable when the crystallization process is slow enough. To further explore this aspect we perform a series of experiments, where we compare the crystallization kinetics of a pure lysozyme system with that of the corresponding mixture. As a typical example we show the temporal evolution of the lysozyme concentration in the low density phase for the systems with $c_{i^{\prime}, \mathrm{LZ}}^{\text {pure }}=c_{i^{\prime} \mathrm{LZ}}^{\operatorname{mix}}=55 \mathrm{~g} \mathrm{l}^{-1}$ and $c_{\mathrm{NaCl}}=400 \mathrm{mM}$ in Fig. 4. At long enough equilibration times the supernatant concentration of lysozyme of both systems corresponds to the saturation limit, $c_{\mathrm{sn}, \mathrm{LZ}}^{\text {mix }}\left(t_{\mathrm{eq}} \rightarrow \infty\right)=c_{\mathrm{sn}, \mathrm{LZ}}^{\text {pure }}>\left(t_{\mathrm{eq}} \rightarrow \infty\right)=$ $S_{\mathrm{LZ}}$, which demonstrates that crystallization of lysozyme is ultimately not affected by the presence of $\alpha$-lactalbumin. However, while for the pure lysozyme system $c_{\mathrm{sn}, \mathrm{LZ}}^{\text {pure }}=S_{\mathrm{LZ}}$ is reached within $\sim 230$ hours, the crystallization of lysozyme in the mixtures is significantly delayed, the saturation limit reached at $t_{\mathrm{eq}} \approx 1600$ hours. Such delay may also determine stability in biological systems, where the proteins are constantly exchanged, the mixtures remaining homogeneous over any relevant life time of the system.

\section{Conclusions}

Our findings expose several generic pathways to stability of concentrated protein mixtures. At high ionic strength segregative phase separation is suppressed when the partial volume fraction of the component prone to phase separate becomes too small to exceed the critical conditions for phase separation, in addition to being significantly delayed by the presence of the other component.
Most importantly, our work denotes the key role of the salt concentration in governing stability. The boundaries to associative phase separation and crystallization delineate the narrow range of ionic strength at which the counter-ions are neither released from the protein double layer nor confined within the protein crystal phase. This can be understood as ionic strength determines the relative contribution of the entropy of the protein counter-ions to the overall thermodynamics of the system. Our experiments with mixtures of proteins of opposite charge effectively probe this contribution in a twofold manner. While the gain in counter-ion entropy drives associative phase separation at low ionic strength, the loss of counter-ion entropy upon confining the counter-ion within the crystal phase becomes small enough at high ionic strength to allow for crystallization. Both observables - the successive suppression of associative phase separation and the onset of crystallization upon increase of the ionic strength - are measures of the decreasing importance of the counter-ion entropy term to the free energy of the overall system. We believe that such combined measures will serve as a valuable benchmark to develop a thermodynamic description that includes the entropy of the counter-ions as a key parameter in the control of the phase behaviour of protein mixtures.

\section{Acknowledgements}

We thank Wilson Poon and Philip A. Pincus for interesting discussions. Financial support by the Adolphe Merkle Foundation is gratefully acknowledged. I.K.V. is grateful for financial support from the European Union through a Marie Curie Fellowship (FP7-PEOPLE-IEF-2008, Contract No. 236723) and The Netherlands Organization for Scientific research (NWO VENI Grant 700.10.406).

\section{Notes and references}

1 A. Stradner, G. Foffi, N. Dorsaz, G. Thurston and P. Schurtenberger, Phys. Rev. Lett., 2007, 99, 198103.

2 A. Pande, J. Pande, N. Asherie, A. Lomakin, O. Ogun, J. King and G. B. Benedek, Proc. Natl. Acad. Sci. U. S. A., 2001, 98, 6116.

3 O. Galkin, K. Chen, R. L. Nagel, R. E. Hirsch and P. G. Vekilov, Proc. Natl. Acad. Sci. U. S. A., 2002, 99, 8479.

4 A. A. Zinchenko, K. Yoshikawa and D. Baigl, Phys. Rev. Lett., 2005, 95, 228101.

5 J. Sun, Q. Zhang and T. Schlick, Proc. Natl. Acad. Sci. U. S. A., $2005, \mathbf{1 0 2}, 8180$.

6 B. Alberts, A. Johnson, J. Lewis, M. Raff, K. Roberts and P. Walter, Molecular Biology of the Cell, Garland Science, New York, 4th edn, 2002.

7 M. R. Wilkins, R. D. Appel, J. E. Van Eyk, M. Chung, A. Görg, M. Hecker, L. A. Huber, H. Langen, A. J. Link and Y. K. Paik, Proteomics, 2006, 6, 4-8.

8 J. M. Berg, J. L. Tymoczko and L. Stryer, Biochemistry, WH Freeman \& Co, New York, 2002.

9 S. B. Zimmerman and S. O. Trach, J. Mol. Biol., 1991, 222, 599-620.

10 K. R. Albe, M. H. Butler and B. E. Wright, J. Theor. Biol., 1990, 143, 163-195.

11 R. P. Sear and J. A. Cuesta, Phys. Rev. Lett., 2003, 91, 245701.

12 R. P. Sear, J. Phys.: Condens. Matter, 2005, 17, S3587-S3595.

13 J. P. K. Doye, A. A. Louis, I. C. Lin, L. R. Allen, E. G. Noya, A. W. Wilber, H. C. Kok and R. Lyus, Phys. Chem. Chem. Phys., 2007, 9, 2197-2205.

14 J. P. K. Doye, A. A. Louis and M. Vendruscolo, Phys. Biol., 2004, 1, P9-P13.

15 G. P. Gorbenko, V. M. Ioffe and P. K. J. Kinnunen, Biophys. J., 2007, 93, 140-153. 
16 F. Galisteo and W. Norde, Colloids Surf., B, 1995, 4, 389-400.

17 D. E. Kuehner, J. Engmann, F. Fergg, M. Wernick, H. W. Blanch and J. M. Prausnitz, J. Phys. Chem. B, 1999, 103, 1368-1374.

18 P. M. Biesheuvel, S. Lindhoud, R. de Vries and M. A. Cohen Stuart, Langmuir, 2006, 22, 1291-1300.

19 E. Spruijt, A. H. Westphal, J. W. Borst, M. A. Cohen Stuart and J. van der Gucht, Macromolecules, 2010, 43, 6476-6484.

20 P. Retailleau, M. RiesKautt and A. Ducruix, Biophys. J., 1997, 73, 2156-2163.

21 A. S. Parmar and M. Muschol, Biophys. J., 2009, 97, 590-598.

22 S. D. Durbin and G. Feher, J. Cryst. Growth, 1986, 76, 583-592.

23 M. L. Broide, T. M. Tominc and M. D. Saxowsky, Phys. Rev. E: Stat. Phys., Plasmas, Fluids, Relat. Interdiscip. Top., 1996, 53, 6325-6335.

24 P. B. Warren, J. Phys.: Condens. Matter, 2002, 14, 7617-7629.

25 J. D. Schmit and K. A. Dill, J. Phys. Chem. B, 2010, 114, 4020-4027.
26 J. D. Schmit, S. Whitelam and K. Dill, J. Chem. Phys., 2011, 135, 085103 .

27 R. Piazza, J. Cryst. Growth, 1999, 196, 415-423.

28 M. Lund, L. Vrbka and P. Jungwirth, J. Am. Chem. Soc., 2008, 130, 11582-11583.

29 M. Lund and P. Jungwirth, J. Phys.: Condens. Matter, 2008, 20, 494218.

30 J. M. Garcia-Ruiz, J. Struct. Biol., 2003, 142, 22-31.

31 N. E. Chayen, Prog. Biophys. Mol. Biol., 2005, 88, 329-337.

32 N. Asherie, Methods, 2004, 34, 266-272.

33 G. W. He, V. Bhamidi, R. B. H. Tan, P. J. A. Kenis and C. F. Zukoski, Cryst. Growth Des., 2006, 6, 1175-1180.

34 Y. X. Liu, X. J. Wang and C. B. Ching, Cryst. Growth Des., 2010 10, $548-558$.

35 S. Talreja, S. L. Perry, S. Guha, V. Bhamidi, C. F. Zukoski and P. J. A. Kenis, J. Phys. Chem. B, 2010, 114, 4432-4441. 